\title{
Assess of the Value of Semen Examination Items in the Diagnosis of Male Infertility
}

\author{
Xiaowen Wang, Yanan Zhang, Yonghui Liu, Shizong Huang, Zhijun Zang* \\ Department of Infertility and Sexual Medicine, The Third Affiliated Hospital, Sun Yat-sen University, Guangzhou, China \\ Email: *zangzhijun@163.com
}

How to cite this paper: Wang, X.W. Zhang, Y.N., Liu, Y.H., Huang, S.Z. and Zang, Z.J. (2021) Assess of the Value of Semen Examination Items in the Diagnosis of Male Infertility. Yangtze Medicine, 5, $1-11$.

https://doi.org/10.4236/ym.2021.51001

Received: February 19, 2020

Accepted: January 5, 2021

Published: January 8, 2021

Copyright $\odot 2021$ by author(s) and Scientific Research Publishing Inc. This work is licensed under the Creative Commons Attribution International License (CC BY 4.0).

http://creativecommons.org/licenses/by/4.0/

\begin{abstract}
Background: Although semen analysis is a basic method to evaluate male fertility, there was a certain deviation between the semen examination and the actual situation in clinical application. Objective: To evaluate the application value of semen examination items in the diagnosis of male infertility. Method: Males recruited from the Third Affiliated Hospital of Sun Yat-sen University from June 2017 to December 2018 were divided into three groups: normal fertility group, physical examination group (premarital physical examination), and male infertility group. Each group received the following examination such as semen analysis (including semen volume, sperm concentration, progressive motility, total motility, round cell concentration), acrosin activity, and sperm morphology. All the semen analysis items mentioned above were operated according to the WHO laboratory manual for the examination and procession of human semen (Fifth edition) and related documents. Results: The normal fertility group and physical examination group showed significantly higher levels in sperm concentration, progressive motility, total motility, and sperm morphology than those in the male infertility group $(P<0.05)$; both the normal fertility group and the physical examination group have a higher qualified rate in sperm concentration, progressive motility, total motility and sperm morphology than the male infertility group, all $P<0.001$. The percentage of patients having all qualified items in each group was $28.13 \%, 6.67 \%$, and $6.51 \%$ separately. Conclusion: The diagnosis efficiency was higher in sperm concentration, progressive motility, total motility, and sperm morphology than in other semen examination items. A more objective and efficient method of semen examination should be explored to facilitate the diagnoses of male infertility in the future.
\end{abstract}

\section{Keywords}

Male Infertility, Semen Analysis, Application Value 


\section{Introduction}

Male infertility is a very common disease, which seriously affects the family stability and the physical and mental health of patients. Semen analysis is a basic method to evaluate male fertility in the world. Since 1970, the World Health Organization (WHO) has begun to formulate, promulgate and popularize the laboratory manual for the examination and procession of human semen in order to establish standardized methods for human semen examination and strict quality control. Its purpose is to provide consistent standards of semen analysis around the world, to facilitate mutual comparison and epidemiological research, and finally to establish objective normal standards for semen examination items to promote the development of semen examination technology [1].

At present, the WHO laboratory manual for the examination and procession of human semen (Fifth edition) published in 2010 has been widely used all over the world. However, we found that there was a certain deviation between the standard set by the manual and the actual situation in clinical application. Many couples have normal reproduction, but the results of the husband's semen examination are abnormal. Chen Guowu et al. believed that only $70 \%$ of routine outcomes of semen examinations were consistent with actual fertility [2]. Another study demonstrated that $15 \%$ of men with normal semen examination were still diagnosed with infertility [3].

What are the reasons for these phenomena? What is the role of various indicators of semen examination in evaluating male fertility? Which item can reflect semen quality more objectively? There are many studies on these topics, but there are still no clear results. This study analyzed the application value of various semen examination items in the diagnosis of male infertility, and provides a theoretical basis for finding an objective method to evaluate male fertility, by comparing the results of seven items of common semen examination in normal fertility group, premarital physical examination group, and male infertility group, including semen volume, sperm concentration, sperm motility, sperm survival rate, round cell concentration, sperm acrosin activity, normal rate of sperm morphology, etc..

\section{Materials and Methods}

\subsection{Objectives}

Males who underwent semen examination in the Third Affiliated Hospital of Sun Yat-sen University from June 2017 to December 2018 were recruited, including normal fertility volunteers (with spouse pregnant within 12 months), premarital physical examination people, and patients with male infertility (excluding reproductive system infection and female factors) and were divided into three groups: normal fertility group, physical examination group (premarital physical examination), and male infertility group. The infertile man enrolled in the male infertility group should have a female partner who had normal results in their fertility evaluations and did not achieve a clinical pregnancy after 12 
months or more of regular unprotected sexual intercourse. Each group received the following examination such as semen analysis (including semen volume, sperm concentration, sperm motility, sperm survival rate, round cell concentration), sperm acrosin activity, and normal rate of sperm morphology.

\subsection{Detection Method}

\subsubsection{Routine Semen Analysis}

After abstinence for 2 - 7 days, semen was collected by masturbation and liquefied at $25^{\circ} \mathrm{C}$. The semen samples were detected by computer-aided sperm analysis system (CASA). The results were judged according to the WHO laboratory manual for the examination and procession of human semen (Fifth edition).

\subsubsection{Determination of Sperm Acrosin Activity}

By using the kit of Shenzhen Huakang Biotechnology Co., Ltd., the improved Kennedy method was adopted for detection of sperm acrosin activity. Operate in strict accordance with the instructions and with reference to the methods of previous literature reports [4].

\subsubsection{Sperm Morphology Detection}

The modified Papanicolaou staining method recommended by the WHO laboratory manual for the examination and procession of human semen (Fifth edition) was used to detect the sperm morphology. The determination of the results was based on the standards established in the WHO manual.

\subsection{Statistical Method}

SPSS 25.0 software package was used for statistical analysis. The measurement data are represented by $\bar{x} \pm \mathrm{s}$. For variance with normal distribution and homogeneity, One-way analysis of variance was adopted and LSD-t method was used for pairwise comparison. For variance with non-normal distribution or heterogeneity, nonparametric rank sum test was adopted, and Kruskal-Wallis method was used for pairwise comparison. Chi-square test was used to compare two rates: in this paper, the Fisher accurate test was used because the sample size of the normal fertility group was small. The significant level was 0.05 (bilateral), and a $P$ value $<0.05$ was considered statistically significant.

\section{Results}

\subsection{General Information}

A total of 3164 people were enrolled in the present study. We counted the mean and normal rate of each sperm parameter in the three groups, including 32 people in the normal fertility group (24 - 35 years old, averaging 30 years old), 1056 people in the physical examination group ( 25 - 35 years old, averaging 29 years old), and 2076 in male infertility group (25 - 41 years old, averaging 32 years old). Subsequently, we selected people who examined all seven items at the same time to compare the qualified rate of each item in different groups, in- 
cluding 32 people in the normal fertility groups ( 24 - 35 years old, averaging 30 years old), 165 people in premarital physical examination groups (25 - 33 years old, averaging 30 years old), and 215 in male infertility groups (27 - 36 years old, averaging 32 years old).

\subsection{Comparison of the Average of Each Semen Test Item in Three Groups}

We observed the mean of each item and made a pairwise comparison. The difference in semen volume among each group was statistically significant (normal fertility group $3.56 \pm 1.53 \mathrm{ml}$, physical examination group $3.33 \pm 1.50 \mathrm{ml}$, and male infertility group $3.53 \pm 1.90 \mathrm{ml}, P=0.025)$. The examination group had a bigger semen volume than the male infertility group, $P=0.04$ (Figure 1 (a)). The sperm concentration: normal fertility group $(70.76 \pm 31.03) \times 10^{6} \mathrm{ml}$, physical

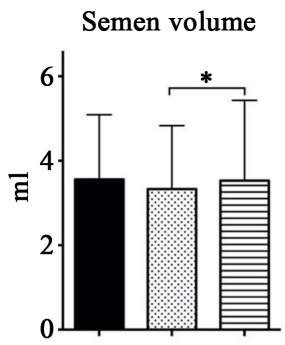

(a)

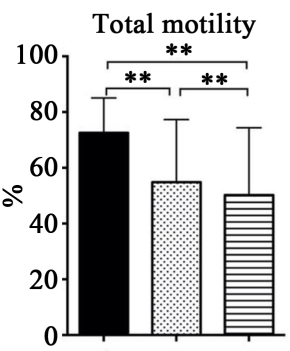

(d)

Sperm morphology

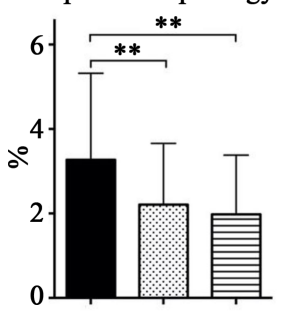

(g)

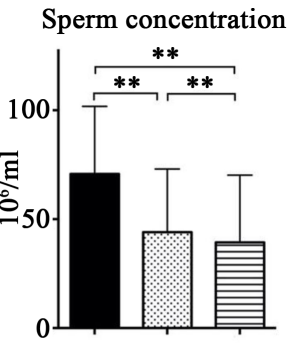

(b)

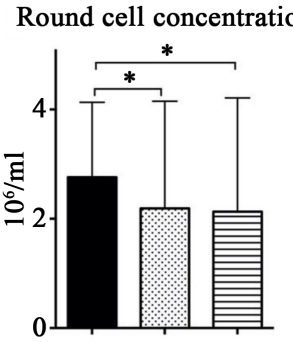

(e)

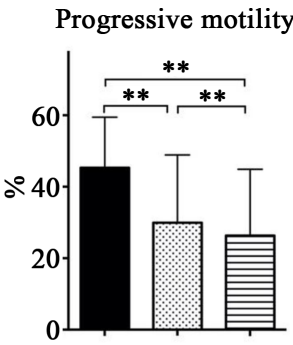

(c)

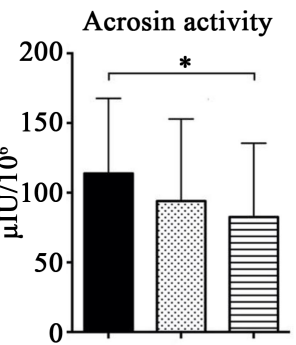

(f)

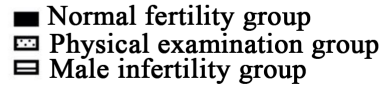

Male infertility group

Figure 1. Comparison of average of each item in three groups. (a) Semen volume in physical examination group was statistically lower than in male infertility group, $P=$ 0.025. Statistical differences were seen among three groups in sperm concentration (b), progressive motility (c), and total motility (d), all $P<0.001$; (e) The round cell concentration was obviously higher in normal fertility group than in the other two groups, $P=0.04$; (f) The acrosin activity in normal fertility group was statistically higher than in male infertility group, $P=0.029$; $(\mathrm{g})$ The sperm morphology was significantly higher in normal fertility group than in the other two groups, $P=0.002$. 
examination group $(44.05 \pm 29.00) \times 10^{6} \mathrm{ml}$, and male infertility group $39.31 \pm$ $30.88 \times 10^{6} \mathrm{ml}$. There were significant differences in sperm concentration among the three groups (all $P<0.001$ ) (Figure $1(\mathrm{~b})$ ). The progressive motility and total motility also showed statistically difference (progressive motility: normal fertility group $45.31 \% \pm 14.17 \%$, physical examination group $29.94 \% \pm 18.96 \%$, male infertility group $26.35 \% \pm 18.54 \%$, all $P<0.001$, Figure 1 (c); total motility: normal fertility group $72.55 \% \pm 12.52 \%$, premarital physical examination group $55.38 \%$ $\pm 22.44 \%$, male infertility group $51.24 \% \pm 24.13 \%$, all $P<0.001$, Figure $1(\mathrm{~d})$ ). As to sperm round cell concentration, there were statistical differences between normal fertility group and the physical examination group $((2.76 \pm 1.37) \times$ $10^{6} / \mathrm{ml}$ vs. $\left.(2.19 \pm 1.96) \times 10^{6} / \mathrm{ml}, P=0.03\right)$, and between normal fertility group and male infertility group $\left((2.76 \pm 1.37) \times 10^{6} / \mathrm{ml} \mathrm{vs.}(2.13 \pm 2.08) \times 10^{6} / \mathrm{ml}, P=\right.$ $0.01)$. The normal fertility group was significantly higher in sperm acrosin activity and in the normal rate of sperm morphology than the male infertility group (perm acrosin activity: $(113.84 \pm 53.81) \mu \mathrm{IU} / 10^{6}$ vs. $(93.98 \pm 58.90) \mu \mathrm{IU} / 10^{6}, P=$ 0.029; normal rate of sperm morphology: $(3.27 \pm 2.05) \%$ vs. $(1.98 \pm 1.40) \%, P<$ $0.001)$. There were also statistical differences in the normal rate of sperm morphology between normal fertility group and physical examination group ((3.27 \pm 2.05)\% vs. (2.21 \pm 1.45$) \%, P=0.002)$ (Figure 1(f) \& Figure $1(\mathrm{~g})$ ).

\subsection{Comparison of the Qualified Rate of Each Item in Different Groups}

We continued to compare the qualified rate of each item in different groups by using Fisher exact test, and found that there was no statistical difference in the qualified rate of semen volume and acrosin activity among the three groups $(P>$ $0.05)$. As to the items of sperm concentration and sperm survival rate, there was no statistical difference in the qualified rate between the normal fertility group and the physical examination group, but both of them were significantly higher than in the male infertility group (sperm concentration: $\mathrm{X}^{2}=34.789, P<0.001$; sperm survival rate: $\left.\mathrm{X}^{2}=32.071, P<0.001\right)$. In the items of sperm motility and normal rate of sperm morphology, there were significant differences among them (sperm motility: $\mathrm{X}^{2}=37.549, P<0.001$; normal rate of sperm morphology: $\left.\mathrm{X}^{2}=19.332, P<0.001\right)$. As to the round cell concentration item, there was no statistical difference between the normal fertility group and the other two groups. However, there was significant difference between the physical examination group and the male infertility group $\left(\mathrm{X}^{2}=19.404, P<0.001\right)$. These results further indicate that sperm concentration, sperm motility, sperm survival rate and normal rate of sperm morphology might be relatively more efficient in the diagnosis of male infertility. The results are shown in Figure 2.

\subsection{The Numbers of Qualified Items in Each Group}

We compared people who completed all examination items at the same time (including semen analysis, sperm acrosin activity and normal rate of sperm morphology) among each group: normal fertility group $(\mathrm{n}=32)$, physical 


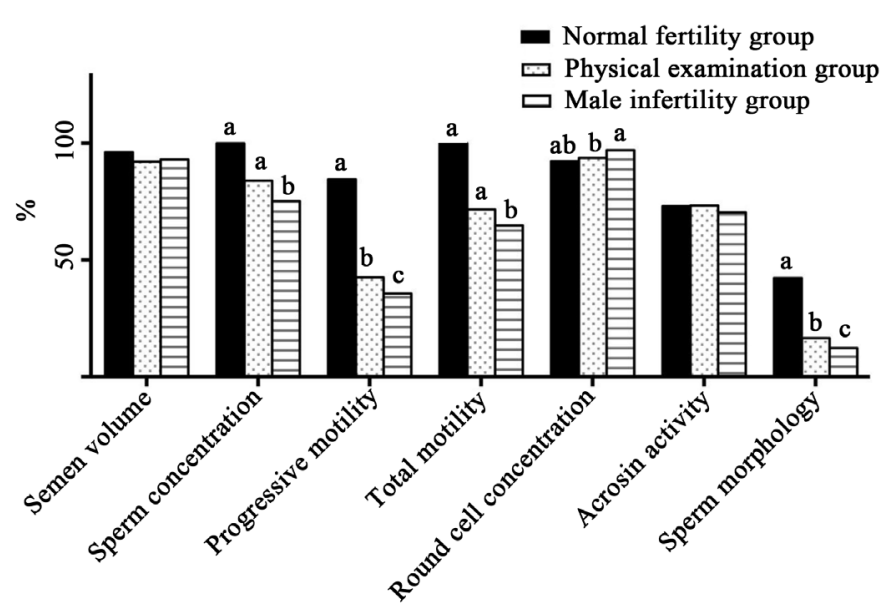

Figure 2. Comparison of the qualified rate of all items in each group. No significant differences in the qualified rate of semen volume and acrosin activity were seen among three groups, $P>0.05$. Both the normal fertility group and the physical group have a higher qualified rate of sperm concentration, progressive motility, total motility and sperm morphology than the male infertility group, all $P<0.001$. Statistical difference was seen in round cell concentration between physical examination group and male infertility group, $P<0.001$.

examination group $(n=165)$ and male infertility group $(n=215)$. It was found that $28.13 \%$ of the people in the normal fertility group had all normal examination items, while only $6.67 \%$ in the physical examination group and $6.51 \%$ in the male infertility group. The results are shown in Figure 3.

\section{Discussion}

There are many factors in semen composition that affect male fertility, including seminal plasma biochemistry, sperm DNA integrity, sperm density, motility and morphology, etc. WHO laboratory manual for the examination and procession of human semen (2010) provides a variety of methods for evaluating semen quality, and establishes a reference range. However, these items cannot provide clinicians with an accurate prediction of male fertility. This is because that there are other factors affecting sperm-egg binding besides semen quality. At first, WHO took sperm concentration $>20 \times 10^{6} / \mathrm{ml}$, motility $>50 \%$, and the normal rate of sperm morphology $>50 \%$ as the normal standard. These results were based on the work of Macleod in the 1950s [5]. In recent years, WHO counted the research data of more than 4500 men from 14 countries who had given birth within 12 months, and used the unilateral 5\% confidence interval to establish the latest reference ranges of semen parameters: semen volume $1.5 \mathrm{~mL}(1.4-1.7$ $\mathrm{mL})$, sperm concentration $15 \times 10^{6} / \mathrm{mL}\left[(12-16) \times 10^{6} / \mathrm{Ml}\right]$, sperm motility $32 \%$ (31\% - 34\%), sperm survival rate 40 (38\% - 42\%), normal sperm morphology rate $4 \%(3 \%-4 \%)[1]$. WHO recommended that the criteria above should be combined with the results of the fertility test of the female spouse to comprehensively assess the couple's fertility status. Although having provided many related semen examination items, and the examination items are divided into basic 


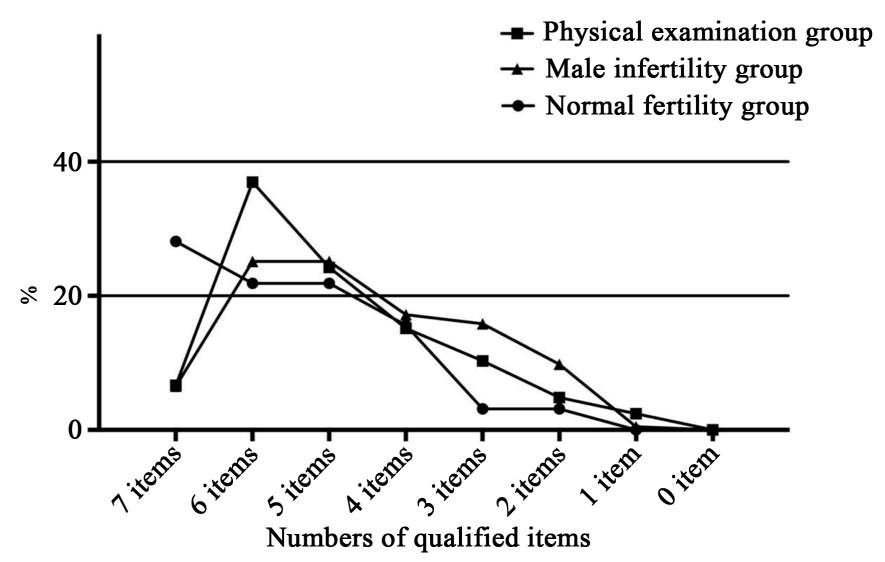

Figure 3. Comparison of qualified items in each group.

items, optional items and scientific research items according to the actual operation, WHO does not give a specific assessment of the importance of each examination item, nor does it explain how clinicians should make an objective and specific diagnosis when facing with several abnormal examination items. In addition, medical conditions vary around the world, and many medical institutions are unable to complete all semen examination items. Therefore, we selected the two most widely used items in the basic items to observe the examination of each item in the diagnosis of male infertility, including: semen analysis (including semen volume, sperm concentration, sperm motility, sperm survival rate, round cell concentration), normal rate of sperm morphology, and the detection of sperm acrosin activity which is widely used in China.

First, we compared the mean of each item among the three groups. Although the average semen volume in the male infertility group was higher than that in the normal fertility group and the premarital physical examination group, only the difference between the male infertility group and the physical examination group was statistically significant. This result indicated that semen volume was not efficient in the diagnosis of male infertility, so it is suggested that except in extreme cases (such as semen volume $<1.0 \mathrm{~mL}$ ), the total number of sperm calculated by the combination of semen volume and sperm concentration in a single ejaculation should be the evaluation indexes. Some studies have proved that the total number of sperm in a single ejaculation is positively correlated with the incubation period of pregnancy [6]. Although it is controversial whether sperm concentration can be used as a biomarker of male infertility, it is still regarded as the most basic examination item for male infertility. Sperm concentration has also been proved to be positively correlated with the incubation period of pregnancy [6]. Our observation results also showed that there was a significant difference in sperm concentration among the three groups. The sperm concentration in the normal fertility group was significantly higher than that in the other two groups, and the physical examination group was also significantly higher than the male infertility group. This outcome indicated that sperm concentration differentiated infertile males from fertile males very well. The evaluation of 
sperm activity is also very important. If the sperm loses its activity, it cannot combine with the egg. In this study, it was found that there were significant differences in sperm motility and survival rate among the three groups. This suggests that the sperm activity index is also accurate in the diagnosis of male infertility. A foreign study continuously observed 501 couples who stopped contraception for 12 months, and found that there was a significant correlation between pregnancy latency and sperm motility [7]. Semen contains non-sperm cells, including epithelial cells from the urogenital tract, as well as white blood cells and immature germ cells, which are collectively referred to as round cells [1]. White blood cells are the main component of round cells [8] [9]. The total number of round cells in semen can reflect the severity of inflammation or the state of spermatogenesis [1]. The average concentration of round cells in the three groups was in the normal range, and the mean in the normal fertility group was slightly higher than that in the other two groups. Therefore, we believe that round cell abnormality can be used as an indicator of genital tract inflammation, but it is not a major factor leading to male infertility. Sperm acrosin is a key enzyme in the process of fertilization, which can dissolve the zona pellucida when the sperm penetrates it, and its activity can directly affect the fertilization [4] [10]. Therefore, acrosin detection is also an important index to reflect the quality of live sperm. This study suggests that the activity of acrosin in normal fertility group and physical examination group is significantly higher than that in male infertility group, so this item is efficient in the diagnosis of male infertility. Sperm morphology detection is an indispensable part of semen quality analysis. At present, most relevant institutions in the world adopt "strict" testing standards. This standard is based on the observation of the morphology of sperm in cervical mucus and Zona pellucida after sexual intercourse, and has been promoted by the 5th edition of WHO semen examination manual [1]. The normal rate of sperm morphology is also related to the incubation period of pregnancy [6]. Our results also showed that the normal rate of sperm morphology in the normal fertility group was significantly higher than that in the male infertility group, but there was no significant difference between the physical examination group and the male infertility group, which also suggests that the ability of this item in differentiating male fertility is relatively good, but weaker than sperm concentration, sperm motility, sperm motility and normal rate of sperm morphology.

Then, we compare the qualified rate of each group. The results showed that in the comparison of the qualified rates in sperm concentration, sperm motility, sperm survival rate and normal rate of sperm morphology, the qualified rates in normal fertility group and premarital physical examination group were significantly higher than that in male infertility group. The qualified rates of sperm motility and normal rate of sperm morphology in the normal fertility group were significantly higher than those in the other two groups. There was no difference in the qualified rates of semen volume and sperm acrosin among all the groups. There was no significant difference in the qualified rate of round cell 
concentration between the normal fertility group and the other two groups, but the qualified rate of round cell concentration in the physical examination group was slightly lower than that in the male infertility group. The above results further suggest that sperm concentration, sperm motility, sperm survival rate and normal rate of sperm morphology are of great significance in the diagnosis of male infertility. At present, numbers of studies have shown that the concentration, motivity and morphological normal rate of sperm can predict "pregnancy incubation period", the evaluation standard of fertility [7] [11]. The WHO laboratory manual for the examination and procession of human semen also lists the above four examination items as the basic items for the diagnosis of male infertility [1]. However, how do we scientifically interpret the above examination items in clinical diagnosis? We counted the normal rate of the above examination items in each group, and found that only $28.13 \%$ of the normal fertility group passed all seven items, which means that $71.87 \%$ of the normal fertility group had at least one abnormal test result. In the physical examination group and male infertility group, $6.67 \%$ and $6.51 \%$ of the population passed all seven results. Therefore, when the clinician is faced with similar semen examination results, they may make different explanations according to previous clinical experience. This also means that the current semen quality testing methods cannot provide a unified standard, so it is impossible to combine the results of various tests to accurately evaluating male fertility.

So how do we scientifically evaluate male fertility in clinical work? At present, there is no unified standard for standardized interpretation of semen test results. Clinicians mainly interpret the semen test data according to their experience, which will inevitably lead to a relatively larger deviation from the actual situation. In addition to the above-mentioned basic items of semen examination, there are other semen factors that affect fertilization. For example, the level of ATP in sperm cells maintains sperm activity [12], sperm mitochondria maintain the normal levels of intracellular calcium ions and reactive oxygen species [13], and the sufficient ADAM3 surface proteins, which is regulated by the other two sperm surface proteins CMTM2A and CMTM2B [14], on the surface of sperm cells are the prerequisites for sperm to pass through cumulus cells and bind to Zona pellucida [15]. At the same time, in addition to semen quality, there are many other factors leading to male infertility, such as the physiological fluctuation of sperm concentration, the mode of insemination (in vivo or in vitro), male health status, female factors and so on. In recent years, an increasing number of researches have proved that the assessment of DNA damage can predict male fertility potential also [16] [17].

Even so, there are several limitations in the present study. First, the sample size in our study is relatively small. The results presented here cannot represent the semen characteristics of men with normal fertility in other districts. Thus a larger sample size, especially a multicenter study, is needed. Second, the present study still did not provide a more objective method to assess human semen. 


\section{Conclusion}

In conclusion, the present study demonstrated that sperm concentration, progressive motility, total motility, and sperm morphology were more efficient in the diagnosis of male infertility than other sperm parameters. The current semen examination methods cannot fully reflect the male reproductive ability; how to correctly interpret the current semen quality test results needs to be further studied. In the future, it is hoped that a more objective and accurate semen evaluation system can be established by the importance rating of existing testing items through multi-center and large samples.

\section{Conflicts of Interest}

The authors declare no conflicts of interest regarding the publication of this paper.

\section{References}

[1] World Health Organization (2010) World Health Organization Laboratory Manual for the Examination and Processing of Human Semen. 5th Edition, Geneva.

[2] Chen, G.W. (1995) Application and Evaluation of Sperm Function Test in Infertile Patients. Reproduction and Contraception, 15, 323.

[3] Lin, M.H., Li, S.H., Lu, C.H., Sun, F.J., et al. (2008) Sperm Chromatin Structure Assay Parameters Are Not Related to Fertilization Rates, Embryo Quality, and Pregnancy Rates in In Vitro Fertilization and Intracytoplasmic Sperm Injection, But Might Be Related to Spontaneous Abortion Rates. Fertility and Sterility, 90, 352-359. https://doi.org/10.1016/j.fertnstert.2007.06.018

[4] Zhang, G., Yang, W., Zou, P., et al. (2019) Mitochondrial Functionality Modifies Human Sperm Acrosin Activity, Acrosome Reaction Capability and Chromatin Integrity. Human Reproduction, 34, 3-11. https://doi.org/10.1093/humrep/dey335

[5] Macleod, J. (1951) Semen Quality in 1000 Men of Known Fertility and in 800 Cases of Infertile Marriage. Fertility and Sterility, 2, 115-139. https://doi.org/10.1016/S0015-0282(16)30482-4

[6] Slama, R., Eustache, F., Ducot, B., et al. (2002) Time to Pregnancy and Semen Parameters: A Cross-Sectional Study among Fertile Couples from Four European Cities. Human Reproduction, 17, 503-515. https://doi.org/10.1093/humrep/17.2.503

[7] Buck Louis, G.M., Sundaram, R., Schisterman, E.F., et al. (2014) Semen Quality and Time to Pregnancy: The Longitudinal Investigation of Fertility and the Environment Study. Fertility and Sterility, 101, 453-462. https://doi.org/10.1016/j.fertnstert.2013.10.022

[8] Cooper, T.G. and Hellenkemper, B. (2010) Improving Precision in the Assessment of Round Cell Numbers in Human Semen. Asian Journal of Andrology, 12, 111-114. https://doi.org/10.1038/aja.2009.15

[9] Barbonetti, A., Bisogno, T., Battista, N., et al. (2017) 2-Arachidonoylglycerol Levels Are Increased in Leukocytospermia and Correlate with Seminal Macrophages. Andrology, 5, 87-94. https://doi.org/10.1111/andr.12283

[10] Cui, Y.H., Zhao, R.L., Wang, Q. and Zhang, Z.Y. (2000) Determination of Sperm Acrosin Activity for Evaluation of Male Fertility. Asian Journal of Andrology, 2, 229-232. 
[11] Bonde, J.P., Ernst, E., Jensen, T.K., et al. (1998) Relation between Semen Quality and Fertility: A Population-Based Study of 430 First-Pregnancy Planners. The Lancet, 352, 1172-1177. https://doi.org/10.1016/S0140-6736(97)10514-1

[12] Wang, C. and Swerdloff, R.S. (2014) Limitations of Semen Analysis as a Test of Male Fertility and Anticipated Needs from Newer Tests. Fertility and Sterility, 102, 1502-1507. https://doi.org/10.1016/j.fertnstert.2014.10.021

[13] Amaral, A., Lourenço, B., Marques, M. and Ramalho-Santos, J. (2013) Mitochondria Functionality and Sperm Quality. Reproduction, 146, 163-174. https://doi.org/10.1530/REP-13-0178

[14] Fujihara, Y., Oji, A., Kojima-Kita, K., Larasati, T. and Ikawa, M. (2018) Co-Expression of Sperm Membrane Proteins CMTM2A and CMTM2B Is Essential for ADAM3 Localization and Male Fertility in Mice. Journal of Cell Science, 131, pii: jcs221481. https://doi.org/10.1242/jcs.221481

[15] Cho, C. (2012) Testicular and Epididymal ADAMs: Expression and Function during Fertilization. Nature Reviews Urology, 9, 550-560.

https://doi.org/10.1038/nrurol.2012.167

[16] Santi, D., Spaggiari, G. and Simoni, M. (2018) Sperm DNA Fragmentation Index as a Promising Predictive Tool for Male Infertility Diagnosis and Treatment Management-Meta-Analyses. Reproductive BioMedicine Online, 37, 315-326.

https://doi.org/10.1016/j.rbmo.2018.06.023

[17] Elbardisi, H., Finelli, R., Agarwal, A., Majzoub, A., Henkel, R. and Arafa, M. (2019) Predictive Value of Oxidative Stress Testing in Semen for Sperm DNA Fragmentation Assessed by Sperm Chromatin Dispersion Test. Andrology.

https://doi.org/10.1111/andr.12743 